\title{
NUREKSAIN KAANUTAN SESURATAN AKSARA BALI NGANGGEN NOTO FONT LAN APLIKASI WANTUAN PAPAN KETIK MULTILING O KEYBOARD
}

\author{
I Made Dwi Antara Jaya', I Kt Paramarta', I.B. Rai ${ }^{2}$ \\ Program Studi Pendidikan Bahasa Bali \\ Universitas Pendidikan Ganesha \\ Singaraja, Indonesia \\ e-mail: \{antarajaya18@yahoo.com, ketut.paramarta, \\ bagus.rai\}@undiksha.ac.id
}

\begin{abstract}
KUUB
Tetilikan puniki matetujon mahbahang (1) Paletan-paletan nyurat aksara Bali nganggen Noto Font ring aplikasi Multiling O Keyboard, (2) Kaanutan sesuratan aksara Bali ring uger-uger pasang lan wangun ring aplikasi Multiling $O$ Keyboard (3) Kaluwihan lan kakirangan Noto Font lan aplikasi Multiling O Keyboard. Jejering tetilikan puniki Noto Font lan aplikasi wantuan Multiling $O$ Keyboard. Panandang tetilikan puniki kaanutan sesuratan pasang lan wangun aksara Bali sakadi wangun, genah, sukat sane nenten nganutin pasang aksara Bali. Kramaning tetilikan sane kaanggen kramaning pratiaksa lan kramaning dokumentasi. Data tureksa sane kaanggen indentifikasi data, reduksi data, klasifikasi data, interpretasi data lan panyutetan. Pikolih tetilikan minakadi (1) paletan nyurat aksara Bali nganggen Noto Font lan aplikasi Multiling O Keyboard sane kadownload lan tata cara nganggen aplikasi marupa nlatarang panujuh nyurat aksara Bali ring aplikasi (2) Kaanutan sesuratan aksara Bali ring uger-uger pasang lan wangun saking aplikasi Multiling $O$ Keyboard, minakadi sesuratan genah, wangun, lan sukating aksara Bali sane nenten manut ring uger-uger pasang lan wangun aksara Bali (3) Kaluwihan Noto Font lan aplikasi prasida nyurat online, marupa media sosial, taler kakirangannyane wantah prasida nganggen ring HP OS android versi 6.0 munggah lan nenten prasida nyurat offline.
\end{abstract}

Kruna Jejaton: Noto Font, aplikasi aksara Bali

\begin{abstract}
This study aims to explain (1) the steps to write Balinese script using the Noto Font in the Multiling O Keyboard application, (2) the truth of Balinese script writing on the rules of pairs and builds in the Multiling O Keyboard application (3) the advantages and disadvantages of the Noto Font application Multiling $\mathrm{O}$ Keyboard. The subject of this research is the Noto Font and Multiling $O$ Keyboard application. The object of this study is the truth of the writing of the pairs and Balinese scripts such as the shape, placement, size that is not in accordance with the rules of Balinese script. The research method used in this study is the pre-trial method and the documentation method. The data analysis used in this study is data identification, data reduction, data classification, data interpretation and conclusions. The results of this study are (1) Steps to write Balinese script using the downloaded Noto Font and Multiling $O$ Keyboard application and how to use it such as explaining the instructions for writing Balinese script (2) Balinese script writing truths from the pairs rules and builds on the application Multiling O Keyboard, in the form of writing placement, shape and size of Balinese script that is
\end{abstract}


not in accordance with the rules of the Balinese script and building. Android OS version 6.0 is fast and can't write offline or without the internet.

Keywords: Noto Font and application Aksara Bali

\section{PURWAKA}

Basa Bali madue linggih pinaka basa ibu ring Bali lan rumaket pisan paiketannyane sareng kahuripan agama miwah budaya. Yening iketang ring bidang budaya, basa Baline sering kabaos pinaka akahnyane. Tegesipun, yening pacang nureksa budaya Bali, wantah basa Bali sane pinih utama kanggen sarana. Basa Bali nenten dados laliang saha patut kapiara olih krama mangda sayan nglimbak, lan paripurna. Sajeroning ngupapira budaya mangda samian krama urati, minakadi Pemerintah Daerah Provinsi Bali ngamedalang Peraturan Daerah No. 3 Warsa 1992 indik basa, aksara lan sastra Bali. Perda puniki negesang mungguing basa Bali patut kaplajahin sareng sisiane pinaka mata pelajaran muatan lokal wajib saking tingkat SD rauh ring tingkat SMA/SMK. Kawentenan sakadi puniki patut pisan ring pidabdab pemerintah pusat sane negesang mangda ngupapira basa daerah, sakadi kasinahang ring Pasal 36 UUD 1945. Sujatine punika sane dahat mabuat pacang tetep ngukuhang lan ngajegang kawentenan adat lan budaya Bali kantos mangkin.

Uratian sane lianan saking Pemerintah Daerah Provinsi Bali ngenenin indik Bahasa, Aksara lan Sastra Bali sampun akeh lan becik pisan nyaratang mangda pengangkatan tenaga kontrak penyuluh basa Bali ring soang-soang desa pakraman ring Bali. Kawentenan puniki mawinan ring Indonesia sampun makeh basa-basa daerah sane sampun ical sakadi ring basa Mawes (Papua), basa Piru, basa Tandia.

Panglimbak basa Bali manut sejarah ipun yening nganutin iusan saking basa sane lianan sane ngranjing ring kosa basa Bali kakepah dados 3 inggih punika, (1) Kawentenan basa Bali sane keni iusan kosa basa Sansekerta sane kabaos basa Bali Kuna, (2) Kosa basa Bali keni iusan saking kosa basa Jawa Kuna, (3) Basa Bali nenten ja wantah madue iusan saking basa Sansekerta lan basa Jawa Kuna manten, nanging basa Bali keni iusan saking basa Indonesia, basa Cina, basa Inggris lan basa duranegara sane lianan (Bawa, 2002).

Basa, aksara lan sastra Bali pinaka dados tetamian saking panglingsir sane saling mapaiketan. Aksara Bali nenten prasida lempas saking basa Bali, mawinan aksara pinaka cihna basa Bali sane kawigunayang ring kahuripan krama adat Bali lan aksara Bali madue paiketan sareng Agama Hindu.

Sane kabaos aksara inggih punika ceciren utawi gegambaran suara sane kawetuang olih I manusa sane wenten ring kertas utawi media sane tiosan (Tinggen, 1993), minakadi batu, lontar, batu miwah sane tiosan. Kawentenan aksara Bali pinaka silih sinunggil aksara saking daerah ring Indonesia sane kantun kapiara olih krama Bali nyantos mangkin. Yening maosang sejarah aksara Bali, aksara Bali mawit saking aksara India sane mawasta Aksara Karosti, nglantur nedunang Aksara Brahmi, saha nglimbak ring India Utara metu Aksara Dewanegari sane kaanggen nyuratang basa Sansekerta, tur ring India Selatan wenten Aksara Pallawa. Panglimbak aksara Dewanegari miwah Pallawa ring Indonesia nganutin panglimbak agama Hindu miwah Budha. Mawit saking panglimbak aksara Dewanegari miwah Pallawa puniki mawetu aksara Kawi utawi aksara Indonesia Kuna. Saking aksara Kawi kasuen-suen ngawetuang aksara Jawi miwah aksara Bali, taler dados aksara-aksara tiosan sane mangkin wenten ring Indonesia (Dinas Kebudayaan Provinsi Bali, 2002). Aksara ring Bali kakepah dados kalih inggih punika aksara Bali latin lan aksara Bali. Aksara latin puniki sering kaanggen nyurat ngeninin indik sane masifat modern, imbanyane sakadi nyurat pidarta, puisi, naskah drama, sarana bacaan sisia, miwah sane lianan. Lianan ring aksara latin, basa Bali taler kasurat ngangge aksara Bali. 
Aksara Bali puniki sering kaanggen nyurat ngeninin indik sane masifat purwa (tradisional), imbanyane sakadi nyurat ring lontar, wariga, pipil, miwah sane lianan.

Panglimbak basa lan aksara Bali ring aab globalisasi sane sakadi mangkin sampun arang pisan, mawinan akeh krama Bali utamannyane para yowana sane maosang mlajah basa Bali punika meweh tur kasengguh kuno, napi malih mlajahin indik aksara Bali nenten ngresep. Kawentenan sakadi puniki ngawinang sayan sue aksara Bali sayan rered tur nyansan ical, mawinan nenten wenten sane urati. Sangkaning pikobet punika akeh utsaha-utsaha mangda nincapang kawagedan indik aksara Bali sakadi ngawigunayang kawentenan sane kasengguh teknologi.

Kawentenan teknologi (IPTEK) madue akeh kawigunan ipun sakadi ngawinang jadma elah makarya, elah nglimbakang gatra, taler pinaka sarana anggen nginutin aab sakadi mangkin sane kabaos instan lan samian yusa uning nganggen teknologi, silih sinunggilnyane HandPhone (HP) lan laptop. Ngawigunayang teknologi pinaka strategi kaanggen nincapang para yowanane mlajahin indik aksara Bali mangda nincapang rasa bangga tekening aksara Bali sane kasengguh kuna. Ring panglimbak nyurat aksara Bali dumun wantah ring media lontar, batu prasasti, miwah sane lianan. Nanging ring aab teknologi informasi sakadi mangkin sane sampun nglimbak, media nyurat aksara Bali prasida kasurat berbasis komputer utawi $h p$ marupa karakter aksara (font). Kawentenan Font aksara Bali sane sampun nglimbak ring krama Bali inggih punika, Bali Simbar, Bali Galang, JG Aksara Bali, Aksara Bali, Tantular Bali, Lilitan, Geguratan, Ian Noto Sans Balinese. Kawentenan samian font aksara Bali punika malian-lianan sane madue kaluwihan lan kakirangan (Corner, 2015).

Program pengetikan aksara Bali (komputerisasi aksara Bali) sane sampun wenten inggih punika ngawigunayang Font Bali Simbar. Nyantos kawentenan Jurusan Pendidikan Bahasa Bali silih sinunggil jurusan ring Fakultas Bahasa dan Seni ring Universitas Pendidikan Ganesha sane pacang calon guru pendidik basa Bali sane professional lan uning ring kawentenan teknologi sane anyar (up to date) madue mata kuliah sane mawasta komputerisasi aksara Bali pinaka mata kuliah kawagedan (keahlian). Komputerisasi inggih punika kawentanan nguah (sistem, perangkat) mangda prasida kaanggen ring komputer utawi nguah (informasi) sane prasida kasimpen olih komputer (Paramarta, 2016).

Font Noto Sans Balinese inggin punika font sane prasida kaanggen ring jaringan internet lan prasida kaangen ring silih sinunggil device HP (MEUI Redifini Android, 2015). Noto Font pinaka font aksara Bali sane kalimbakang olih Google Inc. sane mastandar Unicode. Font puniki berbasis open source sane sampun kalimbakang saking lisensi SIL Open Font License, Version 1.1 (Google Noto Font, 2014). Font aksara Bali kaoprasiang ngganggen wantuan papan ketik Multiling O Keyboard sane marupa papan ketik digital saha nganggen jaringan internet.

Aplikasi papan ketik Multiling $O$ Keyboard puniki wenten ring Play Store Android sane prasida ka dowload gratis. Kaluwihan aplikasi Multiling O Keyboard ri sajeroning aplikasi sane lianan inggih punika aplikasi puniki prasida kawigunayang ri sajeroning browser, HP (sistem oprasi Android 6.0 munggah), internet web (URLs, HTML, XML, CSS, JSON), lan ring aplikasi Multiling $O$ Keyboard akeh madue kirang langkung 200 aksara (font). Nanging, wenten kakirangan aplikasi puniki ngenenin indik aksara Bali minakadi wenten sesuratan aksara sane durung nganutin uger-uger pasang lan wangun aksara Bali sakadi genah, wangun, sukatnyane. Imbanyane wenten aksara sane genahnyane kari iwang silih sinunggilnyane cecek lan pepet punika matumpuk, taler wenten wangun (bentuk) aksara sane iwang sakadi gantungan aksara ra pateh wangunnyane sakadi gantungan wa. Ring tetilikan puniki panilik meled nilikin indik kaiwangan pasang lan wangun aksara Bali.

Madasar antuk napi sane sampun kabahbahang, panilik meled nglaksanayang tetilik ngenenin indik "Nureksain Kaanutan Sesuratan Aksara Bali nganggen Noto Font 
Ian Aplikasi wantuan Papan Ketik Multiling O Keyboard".

\section{KRAMANING TETILIK}

Manut Wendra (2014: 31), kramaning tetilik inggih punika dudonan sane kalaksanayang ri tatkala nyawis pikobet sane kalaksanayang. Kramaning tetilik punika kakepah dados 5 inggih punika, (1) palihan tetilik, (2) jejering lan panandang tetilik, (3) parikrama mupulang data, (4) piranti tetilik, (5) data tureksa.

Palihan tetilik inggih punika tata utsaha sane kaanggen ngamargiang rerawatan sumangdane sane nilikin prasida molihang data sane becik (valid) (Arikunto, 2006: 16). Tetilik sane kanggen ring sajeroning tetilik puniki wantah palihan tetilik deskriptif kualitatif, inggih punika tetilikan sane ngwedarang data sane kapolihang nganggen kruna-kruna. Deskriptif kualitatif punika kaanggen santukan patut sakadi wangun tetilik puniki, sane nyihnayang aksara Bali Noto Font sane wenten ring Aplikasi Multiling $O$ Keyboard lan nureksain kaanutan sesuratannyane mangda nganutin pasang aksara Bali. Jejering inggih punika aran, jadma, genah tetilikan sane pinaka pikobet ring sajeroning tetilik (Wendra, 2013: 32). Ring tetilik puniki sane dados jejering tetilik inggih punika Noto Font lan Aplikasi wantuan Multiling $O$ Keyboard. Sane dados panandang tetilik ring tetilikan puniki inggih punika kaanutan sesuratan pasang lan wangun aksara Bali sakadi wangun, genah, sukat sane nenten nganutin pasang aksara Bali.

Sugiyono (2013: 308) maosang mupulang data inggih punika pahan sane mautama ring sajeroning tetilik santukan tetujon utama saking tetilik inggih punika ngamolihang data sane pinih jangkep. Luihnyane data kacutetang saking piranti sane kaanggen mupulang data. Kramaning sane kaanggen mupulang data ring tetilik puniki inggih punika kramaning pratiaksa lan kramaning dokumentasi. Pratiaksa inggih punika tata cara ngamolihang data antuk mraktiaksa lan nyatet data sane pacang kapupulang. Sadurung jagi nilikin indik kawentenan wangun sane iwang ri sajeroning Aplikasi
Multiling O Keyboard, patut nglaksanayang pratiaksa indik kawentenan pupulan Aplikasi. Pratiaksa kalaksanayang mangda prasida ngamolihang aplikasi sane pacang kaanggen tetilik. Pratiaksa pinaka dudonan kapertama sane kalaksanayang sadurung ngamolihang aplikasi sane patut kanggen tetilik. Iriki kapolihang Aplikasi Multiling $O$ Keyboard sane pacang kaselehin. Kramaning dokumentasi inggih punika data sane kanggen tetilik kapolihang saking cakepan-cakepan (Arikunto, 2005: 45). Kaluihan mupulang data antuk dokumentasi inggih punika nenten akeh nelasang prabeya miwah galah. Kramaning puniki kaanggen ri tatkala mupulang data indik pasang lan wangun aksara Bali sane iwang ring Aplikasi. Pamargi parikrama mupulang data inggih punika kapertama ring aspek penilaian Paletan-paletan nyurat aksara Bali nganggen Noto Font lan aplikasi Multiling O Keyboard ngawigunayang sumber data saking Noto Sans Balinese Regular.ttf. lan Multiling $O$ Keyboard ngangen instrumen utawi piranti Pedoman instal Noto Font lan Multiling $O$ Keyboard, sane kaping kalih ring aspek penilaian Kaanutan sesuratan aksara Bali nganggen Noto Font lan aplikasi Multiling $O$ Keyboard ngawigunayang sumber data saking Salinan Prasasti Basa Bali Kuna (Yumu Pakatahu) ngangen instrumen utawi piranti Pedoman nyurat BBK lan Uger-uger nyurat aksara Bali utawi Pasang Aksara Bali

Piranti sane kaanggen ring tetilik puniki inggih punika piranti pratiaksa lan piranti dokumentasi. Piranti pratiaksa kalaksanayang ring Aplikasi sane pacang kaanggen, ring tetilikan sumangdane uning sapunapi kawentenan wangun aksara Bali sane iwang nenten nganutin pasang aksara Bali ri sajeroning Aplikasi Multiling O Keyboard. Piranti dokumentasi sane ngawigunayang Aplikasi Multiling $O$ Keyboard sane wenten ring Play Store. Piranti sane kanggen nilikin marupa kartu data, kaanggen nureksain indik kawentenan wangun aksara Bali sane nenten nganutin pasang aksara Bali.

Sane kaping untat data tureksa sane kaanggen sajeroning tetilikan puniki kalaksanayang inggih punika indentifikasi data, reduksi data, klasifikasi data, 
interpretasi data, lan panyutetan. Sane kapertama pacang kalaksayang identifikasi data utawi nyelehin data. Iriki patut kalaksanayang tetilik wangun aksara sane iwang ri sajeroning aplikasi mangda prasida ngamolihang data-data sane kaaptiang. Sane kaping kalih Reduksi data inggih punika ngringkes lan ngrereh sane pinih utama. Data sane kareduksi inggih punika data sane nenten kaanggen duaning nenten manut sareng tetujon tetilik. Data sane kaanggen inggih punika data sane mapaiketan sareng tetujon lan bantang pikobet tetilik. Data punika indik wangun aksara sane iwang ring pasang aksara Bali ri sajeroning aplikasi Multiling O Keyboard. Sane kaping tiga, sasampune data punika kaidentifikasi, salanturnyane pacang kamargiang klasifikasi data, inggih punika mupulang data sane sampun jangkep manut sorohnyane. Klasifikasi data puniki kamargiang manut bantang pikobet ring tetilik. Soroh data punika minakadi wangun, sukat, genah aksara sane iwang saking pasang aksara Bali. Sane kaping pat Interpretasi data inggih punika panampen utawi nlatarang ring pikolih tetilik sane sampun kapupulang nginutin pikobet ring tetilik puniki. Data sane mapaiketan sareng soroh-soroh data sane kaaptiang minakadi wangun aksara sane iwang nenten nganutin pasang aksara Bali punika selanturnyane katampenin lan katlatarang mangda prasida karesepin. Salanturnyane Panyutetan pinaka pidabdab kaping untat ring data tureksa puniki. Panyutetan puniki kalaksanayang manut data lan bukti-bukti sane sampun kapolihang. Ring panyutetan puniki pacang kakaryanin tetingkesan indik data sane sampun kapolihang lan kaselehin ngangge deskriptif kualitatif. Panyutetan sane sampun kapolihang punika pinaka pasaur ring bantang pikobet tetilik. Data-data punika manut kawentenan wangun aksara Bali sane iwang ri sajeroning Aplikasi sane sampun kapupulang lan mangkin kacutetang. Pakaryan nyutetang puniki nenten ja wantah apisan, nanging kantos prasida ngamolihang panyutetan sane pinih patut.

\section{PIKOLIH LAN TETEPASAN}

Pikolih lan tetepasan saking tiga bantang pikobet, inggih punika (1) paletanpaletan nyurat aksara Bali nganggen Noto Font ring aplikasi Multiling O Keyboard, (2) kaanutan sesuratan aksara Bali ring ugeruger pasang lan wangun ring aplikasi Multiling $O$ Keyboard, lan (3) kaluwihan lan kakirangan aplikasi Multiling $O$ Keyboard. Parindikan inucap kabahbahang ring sor.

Pikolih kapertama indik paletanpaletan nyurat aksara Bali nganggen Noto Font ring aplikasi Multiling O Keyboard. Yening nyurat aksara Bali nganggen aplikasi Multiling $O$ Keyboard mangda madue HP versi Android 6.0 ka atas mangda prasida utawi support nyurat aksara Bali lan mangda nginstal kalih paletan, inggih punika download Noto Font saking Google lan instal aplikasi Multiling $O$ Keyboard ring Playstore. Sane kapertama instal Noto Font, kawentenan font ring situs Google Noto Font sampun akeh pisan saking samian panegara, suku, lan daerah, silih situnggilnyane Font aksara Bali sane mawasta "Noto Sans Balinese" font aksara Bali puniki pinaka font sane prasida nyurat online. Download Noto Font, mangda ka dowload ring situs resmi Google NotoFonthttps://www. Google.com/get/Noto/ sane kaping kalih instal aplikasi, Multiling $O$ Keyboard inggih punika aplikasi papan ketik sane kaanggen ring android rikala nyurat.

Aplikasi Multiling O Keyboard puniki madue fitur sane spesial sakadi ngadukung multibasa utawi lintang saking 200 aksara font wenten ring aplikasi puniki, silih sinunggilnyane Font anggen nyurat aksara Bali. Yening pacang download aplikasi puniki magenah ring Playstore utawi download saking link https://play.Google.com/store/apps/details?i $\mathrm{d}=\mathrm{kl}$.ime.oh\&hl=en US. Wenten makudangkudang tata cara nyurat nganggen aplikasi Multiling $O$ Keyboard sekadi tata cara nyurat aksara wresastra, gantungan, swalalita, pengangge suara, pengangge tengenan, ceciren pepaosan lan nyurat angka.

Pikolih kaping kalih indik kaanutan sesuratan aksara Bali ring uger-uger pasang lan wangun ring aplikasi Multiling $O$ Keyboard. Sajeroning tetilikan puniki panilik 
nganggen 7 prasasti Bali kuno sane kasurat antuk Noto Font lan aplikasi wantuan Multiling $O$ Keyboard. Prasasti-prasasti punika mamurda Sukawana Al, Bebetin Al,
Trunjan Al, Trunjan B, Bangli Pura Kehen

A2, Gobleg Pura Desa I, Angsari A.

Tabel 1. Persentase kaiwangan uger-uger aksara Bali rikala nyurat Prasasti nganggen Noto Font lan aplikasi wantuan Multiling O Keyboard

\begin{tabular}{lcc}
\hline Kaiwangan & Kruna & Persentase \\
\hline Genah Aksara & 508 & $18,9 \%$ \\
Wangun Aksara & 102 & $3,8 \%$ \\
Sukating Aksara & 59 & $2,2 \%$ \\
& & $\mathbf{2 4 , 9 \%}$ \\
Total & $\mathbf{6 6 9}$ & \\
\hline
\end{tabular}

Saking 7 Prasasti Bali kuno ngamolihang 2.677 kruna sane sampun kasurat nganggen Noto Font lan aplikasi wantuan Multiling O Keyboard. Sajeroning nyurat aksara Bali nganggen Noto Font, panilik ngemanggihin wenten sesuratan sane durung manut ring uger-uger sakadi, wangun aksara, genah aksara lan sukat aksara. Panilik ngamolihang 669 kruna sesuratan sane iwang ring uger-uger taler panilik nyorohang data sane nenten manut ring uger-uger genah, wangun lan sukating aksara. Sesuratan aksara Bali nganggen Noto Font sane nenten anut ring uger-uger genah aksara Bali wenten $18,9 \%$, sane iwang ring uger-uger wangun aksara Bali wenten 3,8 \%, lan sane iwang ring uger-uger sukating aksara Bali wenten 2,2 \%. Pikolih data sane ngawinang sesuratan Noto Font iwang sajangkepnyane kabahbahang ring sor.

Kawentenan genah aksara Bali rikala nyurat nganggen Noto Font lan aplikasi wantuan Multiling $O$ Keyboard durung manut sakadi, Gantungan Ja, pangangge suara ulu, panggangge suara suku, pangangge aksara ulu lan surang, pangangge aksara ulu lan cecek, pangangge tenganan cecek, gantungan ca.

Sane kapertama Gantungan Ja ring sesuratan aksara Bali nganggen Noto Font gantungan ja nenten manut ring uger-uger genah pasang aksara Bali santukan gantungan ja magenah ring sor tengen aksara sane kagantungin, sakadi imba sane nenten anut Dananjaya
นควิขวอน sapatutnyane gantungan ja magenah ring sor tengah-tengah aksara sane kagantungin гово gsoul

Sane kaping kalih Pangangge Suara (ulu) ring sesuratan aksara Bali nganggen Noto Font pangangge suara ulu nenten manut ring uger-uger genah pasang aksara Bali, santukan panggangge suara ulu magenah ring duur katengen, sakadi imba sane iwang

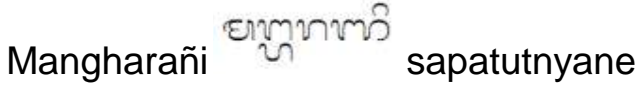
pangangge suara ulu magenah ring duur

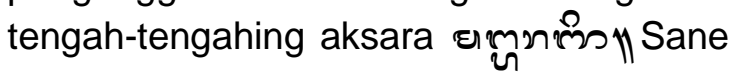
kaping tiga Pangangge suara (suku) ring sesuratan aksara Bali nganggen Noto Font rikala nyurat pangangge aksara sane polih gantungan nenten manut ring uger-uger genah pasang aksara Bali, santukan panggangge suara suku magenah ring aksara sandangan nenten magenah ring aksara sane kagantungin, sakadi

Tanggungan

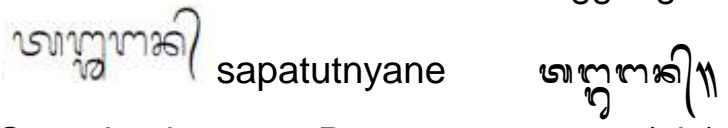

Sane kaping pat Pangangge suara (ulu) lan (surang) ring sesuratan aksara Bali nganggen Noto Font pangangge aksara ulu lan surang nenten manut ring ugeruger genah pasang aksara Bali, santukan aksara ulu lan surang genahnyane matumpuk, sakadi Husir ชyฝึ sapatutnyane pangangge 
aksara ulu magenah sesampun surang vgầ Sane kaping lima Pangangge (ulu) lan (cecek) ring sesuratan aksara Bali nganggen Noto Font pangangge aksara ulu lan cecek nenten manut ring uger-uger genah pasang aksara Bali, santukan pangangge aksara ulu lan cecek genahnyane matumpuk tur aksara ceceknyane kacingak saru, sakadi

Kambing अणही sapatutnyane pangangge aksara ulu magenah sesampun cecek rose्योग Sane kaping nem Pangangge tengenan (cecek) ring sesuratan aksara Bali nganggen Noto Font pangangge tengenan cecek nenten manut ring ugeruger genah pasang aksara Bali, santukan pangangge tenganan cecek magenah ring duur tengen aksara sakadi Culung vovy

sapatutnyane

pangangge tengenan cecek magenah ring duur lan tengah-tengah aksara ggrỳ Sane kaping untat Gantungan $\mathrm{Ca}$ ring sesuratan aksara Bali nganggen Noto Font gantungan ca nenten manut ring ugeruger genah pasang aksara Bali santukan gantungan ca nenten magenah ring sor tengah-tengah, sakadi

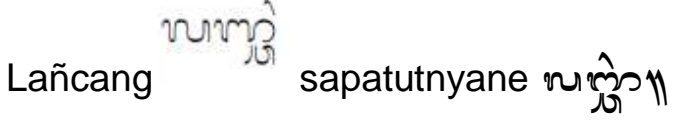

Selanturnyane kaiwangankaiwangan wangun sane wenten rikala nyurat nganggen Noto Font lan aplikasi wantuan Multiling $O$ Keyboard sakadi, Gantungan ra lan gantungan la

Gantungan aksara ra ring sesuratan aksara Bali nganggen Noto Font, gantungan ra nenten manut ring uger-uger wangun pasang aksara Bali santukan gantungan ra sakadi wangun suku kembung imba Satra 2/Yy sapatutnyane gantungan ra wangunnyane

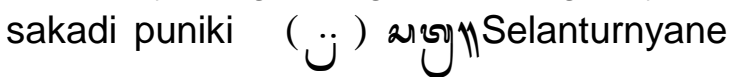
gantungan aksara la ring sesuratan aksara Bali nganggen Noto Font gantungan la nenten manut ring uger-uger wangun pasang aksara Bali, santukan gantungan la sakadi wangun aksara la, imbanyane Pamli vi sapatutnyane gantungan la wangunnyane sakadi puniki (ii ) บध्चीय

Kaiwangan-kaiwangan sukating aksara sane wenten rikala nyurat nganggen Noto Font lan aplikasi wantuan Multiling O Keyboard sakadi, gantungan la lan gantungan nya.

Sane kapertama gantungan aksara la ring sesuratan aksara Bali nganggen Noto Font gantungan la nenten manut ring uger-uger sukating aksara Bali, mawinan gantungan la ring Noto Font sukating aksaranyane apacraken sapatunyane apacraken kirang, imbanyane

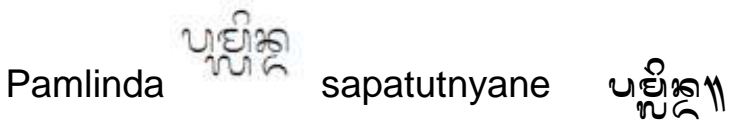

Selanturnyane gantungan aksara nya ring sesuratan aksara Bali nganggen Noto Font gantungan nya nenten manut ring uger-uger sukating aksara Bali, mawinan gantungan nya ring Noto Font sukating aksaranyane apacraken tur genahnyane nenten manut, imbanyane

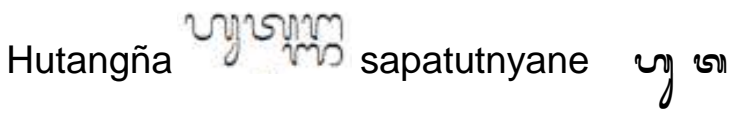
m্

Pikolih sane kaping tiga indik kaluwihan lan kakirangan aplikasi Multiling $O$ Keyboard. Panilik ngalaksanayang tetegar (uji coba) saking aplikasi-aplikasi sane masaih saking Multiling O Keyboard. Wenten makudang-kudang kaluwihan lan kakirangan ring aplikasi.

Aplikasi Multiling $O$ Keyboard madue kaluwihan saking aplikasi sane lianan, Minakadi, kapertama prasida nyurat aksara latin lan aksara sane linanan. Aplikasi Multiling O Keyboard wenten aksara latin pinaka aksara sane mautama rikalaning nyurat aksara-aksara latin, nenten ja madue aksara latin, taler aplikasi Multiling O Keyboard madue akeh aksara-aksara saking suku utawi daerah sane lianan, silih sinunggilnyane aksara Bali. Aplikasi puniki wenten sesuratan aksara Bali sane prasida nyurat online. Sane kaping kalih Aplikasi Multiling $O$ Keyboard prasida nyurat lan kacawen ring 
sekancan aplikasi sane online utawi wenten internet, sakadi, Google Doc, Schoolar, Media Sosial Facebook, Instagram, Twitter, Massanger, Email, WhatsApp.

\section{Aplikasi Multiling $O$ Keyboard} madue kakirangan inggih punika, sane kapertama nenten prasida mengoreksi otomatis kaiwangan uger-uger nyurat. Aplikasi Multiling $O$ Keyboard nenten prasida ngoreksi otomatis kaiwangan nyurat sakadi, nja, nya, tsu, msl. Yening sakadi aplikasi papan ketik sane lian sakadi PaTik madue kaluwihan prasida ngoreksi otomatis. Sane kaping kalih Nenten prasida nyurat ring media offline. Aplikasi Multiling $O$ Keyboard madue kakirangan nenten prasida nyurat lan kawacen ring media offline utawi nenten wenten internet, sakadi aplikasi Word, Excel, Power Point. Sane kaing tiga wantah prasida kaangen ring HP android versi 6.0 kaatas. Aplikasi Multiling $O$ Keyboard wantah prasida kaangen ring HP android sane versi 6.0 kaatas, mawinan yening nganggen versi android 6.0 ka bawah, nenten prasida ngwacen Font aksara utawi aksara Bali, wantah kotak-kotak manten utawi Tofu. Sane kaping pat, wenten kaiwangan ring wangun, sukat lan genah aksara. Sajeroning nyurat nganggen Noto Font lan aplikasi wantuan Multiling $O$ Keyboard, wenten kaiwangan-kaiwangan ring ugeruger pasang aksara Bali, sakadi genah, wangun, lan sukating aksaranyane iwang. Sakadi sane sampun katlatarang ring arep. Sane kaping lima, wantah prasida kaanggen ring HP. Aplikasi Multiling $O$ Keyboard wantah prasida kainstal ring $H P$ android manten, nenten prasida ring Laptop, punika sangkaning wantah prasida ring OS Android kemanten, durung support ring Windows.

Implikasi ring tetilikan puniki inggih punika aksara Bali ring aab sakadi mangkin sampun nglimbak media nyuratnyane, dumun media nyurat aksara Bali wantah ring media tulis lontar, kertas, prasasti lan tembaga. Nanging ring aab aor tanpa wates piranti nyurat aksara Bali sampun nglimbak ring piranti nyurat elektronik, sakadi ring komputer lan HP.
Aksara Bali ring komputer wenten Font Bali Simbar pinaka font sane lumbrah kaanggen rikala nyurat aksara Bali ring Word, Excel, PPT. Yening media nyurat aksara Bali ring HP marupa aplikasiaplikasi papan ketik aksara Bali sakadi PaTik, Multiling $O$ Keyboard sane ngawigunayang aksara saking Noto Font.

Kawentenan panglimbak aksara Bali yening kacingak sayan-sayan becik, mawinan aksara Bali mangkin sampun elah kasurat nganggen HP utawi Komputer. Kawentenan sakadi puniki saking pendidik silih sinunggilnyane Dosen utawi Guru mangda ngawigunayang piranti papan ketik aksara Bali kaanggen media peplajahan mangdane sisia elah mlajahin indik aksara Bali. Lian ring punika kaaptiang aksara Bali sayan-sayan nglimbak nenten ical, mawinan aksara Bali pinaka budaya Bali sane patut iraga jaga kawekas. Pikolih tetilikan puniki kaaptiang kaanggen referensi ngeninin indik mljahin indik pasang aksara Bali lan aplikasi-aplikasi papan ketik sane maaksara Bali.

\section{PAMUPUT}

Saking pikolih miwah tetepasan sane sampun kabahbahang ring ajeng, prasida katingkesan sakadi, sane kapertama paletan-paletan nyurat aksara Bali nganggen Noto Font ring aplikasi Multiling O Keyboard, mangda ngistal kalih paletan inggih punika download Noto Font saking Google lan instal aplikasi Multiling $O$ Keyboard ring Playstore. Sane kaping kalih sajeroning nureksain sesuratan, panilik nyurat 7 prasasti Bali Kuno nganggen Noto Font lan aplikasi wantuan papan ketik Multiling $O$ Keyboard, kapanggihin wenten 2677 kruna sane sampun kasurat nganggen Noto Font nanging wenten kapanggihin 699 sesuratan kruna sane nenten nganutin ring uger-uger pasang aksara Bali sakadi kaiwangan sukating, wangun lan genah. Sane kaping tiga kaluwihan saking aplikasi Multiling $O$ Keyboard inggih punika, prasida nyurat ring aplikasi online sakadi media sosial lan aplikasi puniki prasida nyurat aksara latin.Wenten 
Vol: 6 No: 1, Tahun 2019 e-ISSN : 2599-2627

kakirangan aplikasi Multiling O Keyboard inggih punika nenten prasida nyurat ring media offline sakadi Word, Excel, Ppt, taler aplikasi puniki wantah prasida kaanggen ring HP android versi 6.0 kaatas, wenten kaiwangan-kaiwangan ring sesuratan aksara Bali sakadi ring wangun, genah, sukating aksara.

Piteket kawedarang majeng ring: (1) Majeng dosen miwah guru (tenaga pendidik) tetilikan puniki silih sinunggil piranti pangajahan sane prasida kaanggen nelebin sisia indik mlajah aksara Bali mangda praktis, taler kaaptiang sisia mangda prasida nganggen aplikasi papan ketik Multiling O Keyboard mangda tatas indik aksara Bali lan prasida nglimbakang aksara Bali. (2) Majeng ring panilik sane lianan kaaptiang mangda prasida nglimbakang tetilikan puniki. Panglimbak sane kaaptiang inggih punika mangda nglaksanayang tetilikan sane asoroh, silih sinunggilnyane ngenenin indik aplikasi-aplikasi papan ketik aksara Bali sane lianan sakadi nilikin aplikasi Noto Bali. Indike punika kaaptiang mangda wenten kasandingan pikolih tetilikan sajeroning aplikasi papan ketik aksara Bali sane lianan salanturnyane

\section{KAPUSTAKAAN}

Arikunto. 2006. Prosedur Penelitian. Jakarta: Bina Aksara.

Badan Pembina Bahasa, Aksara dan Sastra Bali. 2002. Kumpulan Makalah Kongres Bahasa Bali V. Denpasar: Balai Bahasa Denpasar.

Bawa, I. W. (2002). Sejarah

Perkembangan Bahasa Bali. Denpasar: Penerbit Universitas Udayana

Corner, N. (2015, October 27). Bringing Balinese to iOS. Retrieved Februari 9,

2019,from:https://norbertlindenberg. com.https://norbertlindenberg.com/2 015/10/bringing-balinese-to-ios/

Dinas Kebudayaan Provinsi Bali. 2002. Pedoman Pasang Aksara Bali.
Denpasar: Dinas Kebudayaan Provinsi Bali.

Dinas Kebudayaan Balai Bahasa. 2009. Kamus Bali-Indonesia Beraksara Latin dan Bali. Denpasar : Balai Bahasa Denpasar.

Google Noto Fonts. (2014, Juli 15). Beatiful and free fonts all language. Retrieved Februari 18, 2019, from Google Noto Fonts:https://www. Google.com/get/N otol

Goris, R. (1954). Prasasti Bali I. Bandung: Masa Baru.

Indrawan, Gede \& Paramarta, I Ketut. 2017. Komputerisasi Transliterasi Teks Latin ke Aksara Bali. Depok: PT Raja Grafindo Persada.

Indrawan, Gede, dkk. 2018. LBtrans-Bot: A Robotic Script Transliteration Script Latin-ke-Bali pada Noto Sans Huruf Bali. Retrieved Februari 24, 2019, from

http://www.iaescore.com/journals/ind ex.php/IJEECS/article/view/13695

Internet Word Stat. (2018, June 30). Internet User in The Word by Region June, 30 2018. Retrieved Februari 11, 2019, from.

https://www.internetworldstats.com:h ttps://www.internetworldstats.com/st ats.htm

MEUI Redefini Android. (2015, July 11). [Tips \& Tutorials] South Asian (Brahmic) and South East Asian Script : Display and Input. Retrieved February 5, 2019, from https://en.miui.com/: https://en.miui.com/thread-1319031-1.html

Paramarta, I Ketut. 2016. Komputerisasi Aksara Bali. Singaraja: Undiksha Press.

Sugiyono. 2013. Media Penelitian Kuantitaif, Kualitatif. Bandung : Sinar Baru.

Tinggen, I Nengah. 1993. Celah-celah Kunci Pasang Aksara Bali. Singaraja: Indra Jaya. 
Unicode. (2018, July 14). Unicode 11.0.0 2018 June 5 (Announcement).

Retrieved 14 Februari 2019, from

:www. Unicode.org:

https://www. Unicode.org/versions/U

nicode11.0.0/

Wendra, I Wayan. 2014. Buku Ajar

Penulisan Karya IImiah. Singaraja:

UNDIKSHA

Wikipedia. (2019, Februari 10). Wikipedia

Papan Ketik. Retrieved Februari 10, 2019,

fromhttps://id.wikipedia.org:https://id. wikipedia.org/wiki/Papan ketik 